\title{
Fundamental Groups for Ergodic Actions and Actions with Unit Fundamental Groups
}

By

\author{
Sergey L. GeFTER* and Valentin Ya. GolODETs**
}

\begin{abstract}
Fundamental groups of ergodic dynamical systems with invariant measure are considered. For a given countable subgroup $\Lambda \subset \boldsymbol{R}_{+}^{*}$, an ergodic dynamical system is constructed whose fundamental group is countable and contains $A$. G.A. Margulis and D. Sullivan revealed that the orthogonal group contains a dense countable subgroup with the proprety $\mathrm{T}$. The fact is applied to construct ergodic actions with a unit fundamental group. The group of outer automorphisms of these actions has also been completely calculated. The proofs are based on the results of studies of the properties of action centralizers.

In the Supplement we introduce the concept of a fundamental group for ergodic actions of continuous groups. It will be shown by means of results of the R. Zimmer's rigidity theory that any finite measure-preserving action of the lattice of the simple Lie group, whose real rank is not less 2 , has a unit fundamental group.
\end{abstract}

\section{§ 0. Introduction}

The fundamental group introduced by F. Murray and J. von Neumann [1] is an important algebraic invariant of the type II factor. The recent progress in the fundamental group studies is connected with consideration of $T$ groups [2]. The first work on this problem was made by A. Connes who has proved that the group factor for the $T$-group with infinite conjugancy classes (ICC-groups) has a countable fundamental group [3]. The $\mathrm{II}_{1}$-factor with a countable fundamental group containing $\Lambda$ was constructed for any given countable subgroup $\Lambda \subset \boldsymbol{R}_{+}^{*}$ in [4]. Thus, it was shown that there existed factors of type $\mathrm{II}_{1}$ with different countable fundamental groups.

The present paper deals with the fundamental groups of ergodic dynamical systems with an invariant measure (see Definition 1.1). The properties of a

Communicated by H. Araki, January 7, 1988.

* Kharkov State University, Department of Mechanics and Mathematics, Dzerzhinsky square 4, Kharkov 310077, USSR.

** Institute for Low Temperature Physics and Engineering, UkrSSR Academy of Sciences, Lenin avenue, 47, Kharkov 310164, USSR. 
dynamical system fundamental group which are similar to those of the factor fundamental group are discussed in Section 1. It is proved that the dynamical system generated by the ergodic action of the ICC-group with property $T$ (TICC-group) which preserves a finite measure has a countable fundamental group (see 1.8 and a more general Theorem 1.7). Furthermore, for a given countable group $\Lambda \subset \boldsymbol{R}_{+}^{*}$ we construct an ergodic dynamical system whose fundamental group is countable and contains $\Lambda$ (Theorem 1.10).

The important results are considered in Section 3-5 where the left shiftsetted action of a countable dense subgroup of the compact group is discussed in detail. It is shown that the properties of a centralizer of this action [5] are determining in this case. By using the dense imbedding of the countable $T$ group into the orthogonal one constructed by G.A. Margulis [6] and D. Sullivan [7], we may calculate the groups of outer automorphisms and the fundamental groups of several dynamical systems [8]. The results obtained (see 4.4, 4.9-4.11 and 5.4) can be formulated as follows:

Let $K=S O(n, \boldsymbol{R}), G=S O(n, \boldsymbol{Q}) n \geqslant 5, n \neq 8, \mu$ be a Haar measure on $K$. Consider the action of $G$ on $(K, \mu)$ generated by left shifts, and let $R_{G}$ be a corresponding equivalence relation, $F\left(R_{G}\right)$ its fundamental group, Aut $R_{G}-a$ group of automorphisms, and Int $R_{G}-a$ subgroup of inner automorphisms.

Theorem. $F\left(R_{G}\right)=\{1\}$,

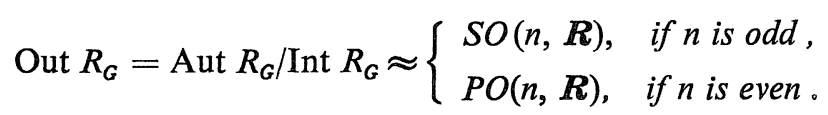

As for Corollaries, see 4.5, 4.7 and 5.5-5.7.

Supplement A presents the construction of a continuum of orbit-nonequivalent ergodic actions for arithmetic groups with the property $T$. In the capacity of the invariant to distinguish between actions, we shall consider the group of outer automorphisms of the factor or equivalence relation generated by the action of the group.

In Supplement B, written together with S.D. Sinelshchikov (see [9]), we introduce the notion of the fundamental group for an ergodic action of a continuous locally compact group. It is shown that any finite measure-preserving action of a semisimple Lie group with a finite center, whose real rank is not less 2, has a unit fundamental group (Theorem B.2). Hence follows triviality of the fundamental group also for actions of lattices of simple Lie groups (Theorem B.3). Note that the problem of existence of the type $\mathrm{II}_{1}$ factor with a unit 
fundamental group remains unsolved.

\section{§1. The Equivalence Relations with Various Fundamental Groups}

Let $(S, \mu)$ be a Lebesgue space with a probability measure $\mu, R$ an ergodic Borel equivalence relation on $(S, \mu)$ with countable orbits which preserves the measure (see [10]). Let us consider a natural equivalence relation $\widetilde{R}=R \times I_{\infty}$ on space $(S \times \boldsymbol{Z}, \mu \times \delta)$, where $\delta(\{n\})=1$ and let Aut $\widetilde{R}$ be its group of automorphisms. If $\theta \in$ Aut $\widetilde{R}$, then there exists $\lambda=\bmod \theta \in \boldsymbol{R}_{+}^{*}$, such that $\tilde{\mu} \circ \theta=\lambda \tilde{\mu}$ [10].

Definition 1.1. The fundamental group of the equivalence relation $R$ is the following subgroup in $\boldsymbol{R}_{+}^{*}$ :

$$
F(R)=\{\bmod \theta: \theta \in \text { Aut } \widetilde{R}\} .
$$

Let us denote the subgroup Aut $\widetilde{R}$ which consists of automorphisms preserving the measure $\mu \times \delta$ by $\operatorname{Aut}_{0} \widetilde{R}$. Then $\mathrm{Aut}_{0} \widetilde{R}$ is a normal subgroup and $F(R) \approx$ Aut $\widetilde{R} /$ Aut $_{0} \widetilde{R}$.

Let $P \subset S$ be a measurable subset of positive measure. Consider the reduced equivalence relation $R \mid P=R \cap(P \times P)$. If $\mu(P)=\mu(Q)$, then $R|P \approx R| Q$ [10, Proposition 3.3]. Hence, for each $\lambda \in(0,1]$ there exists a unique (to within the isomorphism) equivalence relation $R(\lambda) \approx R \mid P$ where $\mu(P)=\lambda$.

As in the case of the factors (see [12]), the properties of the reduced equivalence relation are associated with a fundamental group.

Proposition 1.2. The equivalence relations $R\left(\lambda_{1}\right)$ and $R\left(\lambda_{2}\right)$ are isomorphic if and only if $\lambda_{1} \lambda_{2}^{-1} \in F(R)$.

Proposition 1.3. If $n$ is a natural number, then $\frac{1}{n} \in F(R)$ if and only if $R \approx R \times I_{n}$.

The connection between fundamental group of the equivalence relation and the corresponding factor can be easily established.

Proposition 1.4. Let $M(R)$ be a $I I_{1}$-factor constructed by the equivalence relation $R[11]$ and $F(M(R))$ its fundamental group. Then $F(R) \subset F(M(R))$.

We can apply Proposition 1.4 to prove the countability of fundamental groups of the equivalence relations given by the actions of groups which involve $T$-groups [2].

Let us introduce the following definition. 
Definition 1.5. Let $\Gamma$ and $G$ be countable groups, $\Gamma \subset G, Z(G)$ a center of the group $G$. Let $G$ be called AICC-group with respect to $\Gamma$ if for each $g \notin Z(G)$ the set $\left\{r g \gamma^{-1}: r \in \Gamma\right\}$ is infinite. If this condition is met and $Z(G)=$ $\{e\}, G$ can be called a ICC-group with respect to $\Gamma$. And finally, if $G=\Gamma$ and $G$ is a AICC-group with respect to $\Gamma$, then the group $\mathbb{G}$ can be called a AICCgroup.

Below is given an important example of the AICC-group.

Proposition 1.6. Let $H$ be a connected semisimple Lie group without compact factors, $\Gamma$ be a lattice in $H$ and $G$ the countable subgroup $H$ containing $\Gamma$. Then $G$, is an AICC-group with respect to $\Gamma$; besides, assuming $Z(H)=\{e\}$, the $G$ is an ICC-group with respect to $\Gamma$.

Proof. Let us consider $g_{0} \in G$ such that the set $\left\{r g_{0} r^{-1}: r \in \Gamma\right\}$ is finite. Put $\Gamma_{0}=\left\{r \in \Gamma: r g_{0} r^{-1}=g_{0}\right\}$. We can easily observe that $\Gamma_{0}$ is a subgroup of the finite index in $\Gamma$ and therefore $\Gamma_{0}$ is a lattice in $H$. Further, if $Z_{H}\left(\Gamma_{0}\right)$ is a centralizer of $\Gamma_{0}$ in $H$, then $g_{0} \in Z_{H}\left(\Gamma_{0}\right)$. By the Borel theorem of density, however $Z_{H}\left(\Gamma_{0}\right)=Z(H)$ (see 5.1813 ). Thus, $g_{0} \in Z(G)$.

Note that the groups with property $F$ are also the AICC-groups [14, Lemma 4].

Let $\alpha$ be an free ergodic, preserving the finite measure action of the countable group $G$ on the Lebesgue space $(S, \mu)$. Denote the equivalence relation, generated by the action $G$, by $R_{G}$.

Theorem 1.7. Assume that $G$ is a AICC-group with respect to the subgroup of $\Gamma$ possessing the property $T$ [2]. If the restriction of $\alpha$ on $\Gamma$ is ergodic on $(S, \mu)$ and the center $G$ is finite then the equivalence relation $R_{G}$ has a countable fundamental group.

Proof. Consider a crossed product $M=W^{*}\left(L^{\infty}(S, \mu), \alpha, G\right)$. The algebra $M$ is generated by operators $\pi(a)$ and $\lambda_{g}\left(a \in L^{\infty}(S, \mu), g \in G\right)$ the actions of which in the space $L^{2}(S, \mu) \otimes l^{2}(G)$ are given by the following relationships:

$$
\begin{aligned}
& (\pi(a) \xi)(s, h)=a\left(\alpha_{h}(s)\right) \xi(s, h), \\
& \left(\lambda_{g} \xi\right)(s, h)=\xi\left(s, g^{-1} h\right) .
\end{aligned}
$$

In this case there is an equality $\pi\left(\alpha_{g}(a)\right)=\lambda_{g} \pi(a) \lambda_{g}^{*}, a \in L^{\infty}(S, \mu), g \in G$. Let $\lambda(\Gamma)=\left\{\lambda_{\gamma}: r \in \Gamma\right\}^{\prime \prime}, Q=\lambda(\Gamma)^{\prime} \cap M$; we can show that

$$
\operatorname{dim} Q=|Z(G)| \text {. }
$$


Indeed, let $x \in Q, x=\sum_{g \in G} \pi\left(a_{g}\right) \lambda_{g} . \quad$ Then $\alpha_{\gamma}\left(a_{\gamma^{-1}} g_{\gamma}\right)=a_{g}$, since $\lambda_{\gamma} x \lambda_{\gamma}^{*}=x, r \in \Gamma$. Therefore

$$
\left\|a_{\gamma^{-1}}\right\|\left\|_{2}=\right\| a_{g} \|_{2}, \quad \gamma \in \Gamma, g \in G \text {. }
$$

Hence, $a_{g}=0$ for $g \notin Z(G)$ because $\sum_{g \in G}\left\|a_{g}\right\|_{2}^{2}<\infty$. Thus, $x=\sum_{g \in Z(G)} \pi\left(a_{g}\right) \lambda_{g}$. Further,

$$
\alpha_{\gamma}\left(a_{\gamma^{-1}} \gamma\right)=\alpha_{\gamma}\left(a_{g}\right)=a_{g}, \quad \gamma \in \Gamma, g \in Z(G)
$$

and because of the ergodicity of $\Gamma, a_{g} \in C$. We can conclude that

$$
Q=\operatorname{Lin}\left\{\lambda_{g}: g \in Z(G)\right\} .
$$

Thus, the proof of equality (1.1) complete. Besides, the algebra $M$ is a factor since $Z(G)$ acts on $(S, \mu)$ in a nontrivial way. Let $q$ be a minimum projector in the algebra $Q$ (it will be recalled that $|Z(G)|<\infty$, i.e. $\operatorname{dim} Q<\infty$ ). Then the relative commutant $\lambda(\Gamma)_{q}$ in the reduced factor $M_{q}$ is trivial. By using this fact and the $T$-property of the group $\Gamma$, the countability of the $M_{q}$-factor fundamental group can be proved by the same method as in 1.1 [4]. So, the factor $M$ too has a countable fundamental group. According to 1.4, the group $F\left(R_{G}\right)$ is also countable.

Corollary 1.8. The equivalence relation generated by the free ergodic action of the ICC-group with T-property (TICC-group), which preserves the finite measure, has a countable fundamental group.

Example 1.9. Let $G=S L(n, \boldsymbol{Q}), \Gamma=S L(n, \boldsymbol{Z}), n \geqslant 3$. The group $G$ acts by the left shifts on the space $\{0,1\}^{G}$. One can easily observe that each of the assumptions of Theorem 1.7 is fulfilled and therefore, the fundamental group of the equivalence relation $R_{G}$ is countable.

We can show now that there exist ergodic dynamical systems with nontrivial countable fundamental groups (the fact has been proved for factors in [4]).

Let $\Lambda$ be a countable subgroup of $\boldsymbol{R}_{+}^{*}$ and $\lambda_{i} \in(0,1), i=1,2, \cdots$ its generators. Define the measures $\nu_{i}$ on a two-point space:

$$
\nu_{i}(\{0\})=\frac{1}{1+\lambda_{i}}, \quad \nu_{i}(\{1\})=\frac{\lambda_{i}}{1+\lambda_{i}} .
$$

Consider the space $\left(S_{0}, \mu_{0}\right)=\prod_{i}\left(S_{\lambda_{i}}, \mu_{\lambda_{i}}\right)$ where $S_{\lambda_{i}}=\{0,1\}^{N}, \mu_{\lambda_{i}}$ is the measure 
on $S_{\lambda_{i}}$ constructed as the product of measures $\nu_{i}$. Put $(S, \mu)=\prod_{\gamma \in \Gamma}\left(S_{0}, \mu_{0}\right)$ for the TICC-group $\Gamma$. Then the group $\Delta=\oplus \boldsymbol{Z} / 2 \boldsymbol{Z}$ acts coordinate-wise on each space $S_{\lambda_{i}}$. Therefore, there appears the action of $\Delta$ on spaces $\left(S_{0}, \mu_{0}\right)$ and $(S, \mu)$ because $\bigoplus_{i} \Delta \approx \bigoplus_{\gamma \in \Gamma}\left\{\oplus_{i} \Delta\right\} \approx \Delta$. Consider also the left-shift-generated action of the group $\Gamma$ on $(S, \mu)$. Let $R_{\Delta}$ be the equivalence relation generated by the action $\Delta$, Aut $_{\mu} R_{\Delta}$ a subgroup of Aut $R_{\Delta}$ consisting of automorphisms preserving the measure $\mu$, and

$$
\operatorname{Int}_{\mu} R_{\Delta}=\text { Aut }_{\mu} R_{\Delta} \cap \operatorname{Int} R_{\Delta} .
$$

Denote by $R(\Lambda, \Gamma)$ the equivalence relation on $(S, \mu)$ generated by the action of the semidirect product

$$
H=\Gamma \text { S Int } \operatorname{I}_{\mu}
$$

(evidently, $\Gamma \subset$ Aut $_{\mu} R_{\Delta}$ and $\Gamma \cap \operatorname{Int}_{\mu} R_{\Delta}=\{\mathrm{id}\}$ ).

Theorem 1.10. The equivalence relation $R(\Lambda, \Gamma)$ has a countable fundamental group containing the group $A$.

Proof. Let us introduce a $\mathrm{II}_{1}$-factor $P$ which is constructed by the equivalence relation generated by the group $\operatorname{Int}_{\mu} R_{\Delta}$ (see [11]), and the factor $K$ constructed by the action of group $\Delta$. Since $\Gamma \subset$ Aut $_{\mu} R_{\Delta}$ and $\Gamma \subset$ Aut $R_{\Delta}$, the action of $\Gamma$ on $(S, \mu)$ can be extended up to the action through automorphisms on the factor $K$ and $P$. The group $\Gamma$ then will act ergodically on $K$ and hence on $P$. According to the arguments of the proof of Theorem 1.1 [4], the crossed product $M=W^{*}(P, \Gamma)$ has a countable fundamental group. Then we can show that the factor $M$ is isomorphic to a factor constructed by the equivalence relation $R(\Lambda, \Gamma)$. Therefore, the fundamental group of the equivalence relation $R(\Lambda, \Gamma)$ is countable (see 1.4). We can prove that $\Lambda \subset F(R(\Lambda, \Gamma)$ ). If $E \subset S$ and $\mu(E)=\lambda \in \Lambda$ then because of the ergodicity of $H$ there exists a Borel isomorphism $w$ from $S$ on $E$ such that $\mu \circ w=\lambda \mu$ and for almost all $s \in S,(s,(w(s))$ belongs to the equivalence relation $R_{\Gamma(S)}$ generated by the action of a semidirect product of $\Gamma$ by $\Delta$. Let $\left(s_{1}, s_{2}\right) \in R(\Lambda, \Gamma)$. Then $s_{2}=v\left(s_{1}\right)$ for a particular automorphism $v$ of the group $H$. Therefore, $w\left(s_{2}\right)=w\left(v\left(s_{1}\right)\right)=\left(w v w^{-1}\right)\left(w\left(s_{1}\right)\right)$. But $\left(w\left(s_{1}\right), w\left(s_{2}\right)\right)=\left(w\left(s_{1}\right),\left(w v w^{-1}\right)\left(w\left(s_{1}\right)\right)\right) \in R_{\Gamma \Subset \Delta}$ and besides $w v w^{-1}$ preserves the measure $\mu$. Therefore, $\left(w\left(s_{1}\right), w\left(s_{2}\right)\right) \in R(\Lambda, \Gamma)$ and thus $\left(w\left(s_{1}\right), w\left(s_{2}\right)\right) \in$ $R(\Lambda, \Gamma) \mid E$ because $w(S)=E$. Therefore, $w$ realizes the isomorphism of the equivalence relations $R(\Lambda, \Gamma)$ and $R(\Lambda, \Gamma) \mid E$, i.e. $\lambda=\mu(E) \in F(R(\Lambda, \Gamma))$. 
Corollary 1.11. There exist dynamical systems with different countable fundamental groups.

\section{§ 2. Topological Properties of the Group of Outer Automorphisms of the Equivalence Relation}

In this section some topological properties of the groups of automorphisms of factors and dynamical systems are considered.

Let $(S, \mu)$ be a Lebesgue space with finite or $\sigma$-finite measure $\mu, A=$ $L^{\infty}(S, \mu), \alpha$ be a free ergodic, preserving measure action of the countable group $G$ on the $(S, \mu)$, and let $R_{G}$ be a equivalence relation, generated by the action $G$, Aut $R_{G}-$ a group of all its automorphisms, and Int $R_{G}-$ a subgroup of inner automorphisms [10]. Denote the groups of cocycles and coboundaries of the ation of $G$ with the values in the group $\boldsymbol{T}$ by $Z^{1}(G, U(A))$ and $B^{1}(G, U(A))$, respectively.

Consider a crossed product $M=W^{*}(A, \alpha, G)$ and put

$$
\begin{aligned}
& \text { Aut }(M, A)=\{\theta \in \text { Aut } M: \theta(\pi(A))=\pi(A)\}, \\
& \text { Int }(M, A)=\text { Aut }(M, A) \cap \operatorname{Int} M, \\
& Z(M, A)=\{\theta \in \text { Aut } M: \theta(\pi(a))=\pi(a), a \in A\}, \\
& B(M, A)=Z(M, A) \cap \operatorname{Int}(M, A) .
\end{aligned}
$$

Lemma 2.1. (i) $Z(M, A) \approx Z^{1}(G, U(A))$;

(ii) $B(M, A) \approx B^{1}(G, U(A))$;

(iii) Aut $(M, A) Z(M, A) \approx$ Aut $R_{G}$;

(iv) Int $(M, A) B /(M, A) \approx$ Int $R_{G}$.

See the proof in [11].

We can assign the topology on the group Aut $M$ by using the pre-base of unity neighbourhoods [15]:

$$
\bigcup_{\psi, \varepsilon}=\{\theta \in \text { Aut } M:\|\psi \circ \theta-\psi\|<\varepsilon\}, \quad \psi \in M_{*}, \varepsilon>0 .
$$

For this topology, Aut $M$, its closed subgroups Aut $(M, A)$ and $Z(M, A)$ and the group Aut $R_{G}$ which is identified with Aut $(M, A) / Z(M, A)$ and provided with a quotient topology are Pollish groups.

Lemma 2.2. Let $G$ be a AICC-group with respect to the ergodic subgroup with the property $T$ and the center $G$ be finite. Then the group Int $R_{G}$ is a closed 
subgroup Aut $R_{G}$ and therefore the group of outer automorphisms

Out $R_{G}=$ Aut $R_{G} /$ Int $R_{G}$ is a Pollish one.

Proof. According to the arguments adduced when proving Proposition $6(b)$ in [14], the group $G$ has the property F. Moreover, its action is strongly ergodic (see [16]). Hence, on the basis of Theorem 13 [14], the factor $G$ is full [17]. Thus, Int $M$ and therefore, $\operatorname{Int}(M, A)$ and $B(M, A)$ are closed subgroups of Aut $M$. Then the statement of the Lemma follows from 2.1.

Hence, for the actions which satisfy the conditions of Lemma 2.2 (in particular, for the ergodic actions os TICC-groups), the problem of studying the group of outer automorphisms as a topological group is quite natural. Note that in Section 4 the ergodic actions with compact connected groups of outer automorphisms will be constructed.

The following statement which generalizes Theorem 2.2 [5] is a basis for obtaining subsequent results.

Theorem 2.3. Let $G$ be an ICC-group with respect to the ergodic subgroup $\Gamma$ with the property $T$. Put

$$
C_{\Gamma}=\left\{\theta \in \text { Aut } M: \theta\left(\lambda_{\gamma}\right)=\lambda_{\gamma}, r \in \Gamma\right\}
$$

and let $\varepsilon:$ Aut $M \rightarrow$ Out $M$ be a natural projection. Then, $\varepsilon\left(C_{\Gamma}\right)$ is the open subgroup Out $M$ topologically isomorphic to $C_{\Gamma}$.

Proof. Since the group $\Gamma$ acts ergodically on $(S, \mu)$, then $\left\{\lambda_{y}: r \in \Gamma\right\}^{\prime} \cap$ $M=C$ (see the proof of Theorem 1.7). Denote by $C_{\Gamma}^{M}$ the subgroup Aut $M$ which is algebraically generated by $C_{\Gamma}$ and Int $M$. Then, $C_{\Gamma}^{M}$ is the open subgroup Aut $M$ [5, Theorem 2.2]. Thus, $\varepsilon\left(C_{\Gamma}^{M}\right)$ is the open subgroup Out $M$. Besides, $C_{\Gamma} \cap \operatorname{Int} M=\{\mathrm{id}\}$. Hence, $\varepsilon\left(C_{\Gamma}^{M}\right)=\varepsilon\left(C_{\Gamma}\right)$ is the open subgroup Out $M$, algebraically isomorphic to $C_{\Gamma}$. Now, Out $M$ is a Polish group [18], and $\varepsilon\left(C_{\Gamma}\right)$ its open, and therefore also close, subgroup. It is also obvious that $C_{\Gamma}$ is the closed subgroup Aut $M$. Thus, $C_{\Gamma}$ and $\varepsilon\left(C_{\Gamma}\right)$ are Polish groups and $\varepsilon$ performs their topologic isomorphism [17, Lemma 3.4].

\section{§3. The Equivalence Relation Generated by Shifts of the Countable Dense Subgroup of the Compact Group}

Let $K$ be a compact group, $\mu$ a Haar measure on $K$, and $G$ and $\Gamma$ countable dense subgroups of $K, \Gamma \subset G$. Consider the actions of groups $G$ and 
$K$ on the algebra $A=L^{\infty}(K, \mu)$ generated by left and right shifts, respectively:

$$
l_{g}(a)(k)=a\left(g^{-1} k\right), \quad r_{t}(a)(k)=a(k t), \quad a \in A, g \in G, t \in K .
$$

The automorphisms of space $K$ can be also denoted by $l_{g}$ and $r_{t}$ :

$$
l_{g}(k)=g k, \quad r_{t}(k)=k t^{-1}, \quad t, k \in K, g \in G .
$$

It is evident that the action of $G$ is free and its restriction on $\Gamma$ is ergodic.

Lemma 3.1. Let $\beta: K \rightarrow K$ be a Borel mapping such that $\beta l_{g}=l_{g} \beta$ for all $g \in G$. Then $\beta=r_{t_{0}}$ for a particular $t_{0} \in K$.

Proof. Put $\varphi(k)=k^{-1} \beta(k), k \in K$. Then $\varphi$ is a Borel mapping and by the condition, $\varphi(g k)=\varphi(k)$ for all $g \in G$ and a.a. $k \in K$. Based on this fact and the ergodicity of the action of $G$, one can easily find that $\varphi(k)=t_{0}^{-1}$ for a.a. $k \in K$. Hence, $\beta=r_{t_{0}}$.

This version of the proof was found by S.D. Sinelshchikov.

Consider the crossed product $M=W^{*}(A, l, G)$. Recall that the algebra $M$ is generated by the operators $\pi(a)$ and $\lambda_{g}(a \in A, g \in G)$, where

$$
\lambda_{g} \pi(a) \lambda_{g}^{*}=\pi\left(l_{g}(a)\right) .
$$

Lemma 3.2. If $G$ is a ICC-group with respect to $\Gamma$, and

$$
\theta \in C_{\Gamma}=\left\{\theta \in \text { Aut } M: \theta\left(\lambda_{\gamma}\right)=\lambda_{\gamma}, r \in \Gamma\right\} \text {, }
$$

then the automorphism $\theta$ maps the algebra $A$ into itself, i.e. $\theta \in \operatorname{Aut}(M, A)$.

Proof. Let $\hat{K}$ be a dual space of the group $K$. For every $\sigma \in \hat{K}$ we can choose an orthonormalized basis $\left\{\xi_{i}^{\sigma}: i=1, \cdots, \operatorname{dim} \sigma\right\}$ in the representation space $H_{\sigma}$ and put

$$
\sigma_{i j}(k)=\left(\sigma(k) \xi_{j}^{\sigma} \mid \xi_{i}^{\sigma}\right), \quad k \in K, i, j=1, \cdots, \operatorname{dim} \sigma .
$$

The system $\left\{\sigma_{i j}: \sigma \in \hat{K}, i, j=1, \cdots, \operatorname{dim} \sigma\right\}$ forms an orthogonal basis in $L^{2}(K, \mu)$ with $\sigma_{i j} \in A=L^{\infty}(K, \mu)$.

For $r \in \Gamma$ we have $\sigma_{i j}\left(\gamma^{-1} k\right)=\sum_{p=1}^{\operatorname{dim} \sigma} \sigma_{i p}\left(\gamma^{-1}\right) \sigma_{p j}(k), k \in K$, i.e.

$$
l_{\gamma}\left(\sigma_{i j}\right)=\sum_{p=1}^{\operatorname{dim} \sigma} \overline{\sigma_{p i}(\gamma)} \sigma_{p j} .
$$

Let $\theta \in C_{\Gamma}$ and 


$$
\theta\left(\pi\left(\sigma_{i j}\right)\right)=\sum_{g \in G} \sum_{\rho \in \hat{K}} \sum_{m, n=1}^{\operatorname{dim} \rho} c\left(g ; m, n, \rho ; \sigma_{i j}\right) \pi\left(\rho_{m n}\right) \lambda_{g} .
$$

Since the algebra $A$ is generated by matrix elements $\sigma_{i j}$, it is sufficient to prove that

$$
\theta\left(\pi\left(\sigma_{i j}\right)\right) \in \pi(A), \quad \sigma \in \hat{K}, i, j=1, \cdots, \operatorname{dim} \sigma 。
$$

By using (3.2), the equality

$$
\lambda_{\gamma} \theta\left(\pi\left(\sigma_{i j}\right)\right) \lambda_{\gamma}^{*}=\theta\left(\pi\left(l_{\gamma}\left(\sigma_{i j}\right)\right)\right),
$$

the ICC-property, and the convergence of series (3.3), we can obtain $c\left(g ; m, n, \rho ; \sigma_{i j}\right)=0$ for $g \neq e$ and hence, $\theta\left(\pi\left(\sigma_{i j}\right)\right) \in \pi(A)$.

Denote by $R_{t}$ the automorphism of the factor $M$ given by the relationships

$$
R_{t}(\pi(a))=\pi\left(r_{t}(a)\right), \quad R_{t}\left(\lambda_{g}\right)=\lambda_{g}, t \in K, g \in G .
$$

Besides, the automorphism of $M$ generated by the cocycle $c \in Z^{1}(G, U(A))$ can be denoted by $\theta_{c}$ :

$$
\theta_{c}(\pi(a))=\pi(a), \quad \theta_{c}\left(\lambda_{g}\right)=\pi\left(c_{g}\right) \lambda_{g} .
$$

Proposition 3.3 Let $G$ be a ICC-group with respect to $\Gamma$. If $\theta \in C_{\Gamma}=$ $\left\{\theta \in\right.$ Aut $\left.M: \theta\left(\lambda_{\gamma}\right)=\lambda_{\gamma}, \gamma \in \Gamma\right\}$, then $\theta=\theta_{c} R_{t}$, where $c \in Z^{1}(G, U(A))$ and $t \in K$ are determined unambiguously. In this case $c_{\gamma}=1$ for $r \in \Gamma$.

Proof. In view of Lemma 3.2, $\theta$ determines the automorphism $\beta$ of the algebra $A: \pi \circ \beta=\theta \circ \pi$. By the assumption, $\theta\left(\lambda_{\gamma}\right)=\lambda_{\gamma}$ and so, $\beta l_{\gamma}=l_{\gamma} \beta, \gamma \in \Gamma$. According to $3.1, \beta=r_{t}$ for a particular $t \in K$. Put $\theta_{1}=\theta R_{t}^{-1}$. Then $\theta_{1}(\pi(a))=$ $\pi(a), a \in A$. Therefore there will be $c \in Z^{1}(G, U(A))$ such that $\theta_{1}\left(\lambda_{g}\right)=\pi\left(c_{g}\right) \lambda_{g}$ i.e. $\theta_{1}=\theta_{c}$. Hence, $\theta=\theta_{c} R_{t}$.

Corollary 3.4. If $G$ is a ICC-group, $M=W^{*}(A, l, G)$ then $\{\theta \in$ Aut $M$ : $\left.\theta\left(\lambda_{g}\right)=\lambda_{g}, g \in G\right\}=\left\{R_{t}: t \in K\right\}$.

Theorem 3.5. Let $\Gamma$ have the property $T$ and $G$ be a ICC-group with respect to $\Gamma$. Then

$$
\varepsilon\left(\left\{\theta_{c} R_{\dot{t}}: t \in K, c \in Z^{1}(G, U(A))\right\}\right)
$$

is an open subgroup Out $M$, topologically isomorphic to the semidirect product $K\left(S H^{1} /(G, U(A))\right.$.

Proof. By 2.6 and 3.3, 


$$
\varepsilon\left(\left\{R_{t}: t \in K\right\} @ Z^{1}(G, U(A))\right)
$$

is an open subgroup of Out $M$. Since $G$ is the ICC-group with respect to $\Gamma$, then

$$
\left(\left\{R_{t}: t \in K\right\} @ Z^{1}(G, U(A))\right) \cap \operatorname{Int} M=B^{1}(G, U(A)),
$$

i.e. the groups $K\left(H^{1}(G, U(A))\right.$ and

$$
\varepsilon\left(\left\{R_{t}: t^{\prime} \in K\right\} \subseteq Z^{1}(G, U(A))\right)
$$

are algebraically isomorphic. On the other hand, one can easily observe that $K$ is topologically isomorphic to $\left\{R_{t}: t \in K\right\}$. Furthermore, the action of $G$ is strongly ergodic and $H^{1}(G, U(A))$ is therefore a Pollish group (see 3.2 [16]). From Lemma 3.4 [17] and the continuity $\varepsilon$ it follows the group (3.4) is topologically isomorphic to $K\left(H^{1}(G, U(A))\right.$.

Corollary 3.6. If $G$ is a TICC-group, $M=W^{*}(A, l, G)$, then $\varepsilon\left(\left\{R_{t}: t \in K\right\}\right.$ is an open subgroup of Out $M$ which is topologically isomorphic to $K$.

Consider now in detail a case when the group $K$ is connected First of all, it should be noted that the AICC-property of the group $G$ with respect to $\Gamma$ is readily realized (cf. 1.6).

Proposition 3.7. Let $K$ be a connected compact group, $\Gamma$ and $G$ be countable dense subgroup of $K, \Gamma \subset G$. Then $G$ is a AICC-group with respect to $\Gamma$. But if the center of $K$ is trivial, then $G$ is a ICC-group with respect to $\Gamma$.

Proof. For $g_{0} \in G$ such that the set $\left\{r g_{0} r^{-1}: r \in \Gamma\right\}$ is finite, we can take $\Gamma_{0}=\left\{r \in \Gamma: r g_{0}=g_{0} r\right\}$. One can easily show that $\Gamma_{0}$ is a subgroup of the finite index in $\Gamma$ and so, according to the connectivity of $K, \Gamma_{0}$ is dense in $K$. Hence, $g_{0} \in G \cap Z(K)=Z(G)$.

Lemma 3.8. Let $K$ be a compact group with a finite center, $G$ be a countable dense subtgoup of $K, \widetilde{G}=G / Z(G), \tilde{K}=K / Z(G)$. Condider the equivalence relations $R_{G}$ and $R_{\tilde{G}}$, generated by the actions of $G$ on $K$ and $\tilde{G}$ on $\tilde{K}$, respectively. Then $R_{G} \approx R_{\tilde{G}} \times I_{n}$, where $n=|Z(G)|$ and therefore,

Out $R_{G} \approx$ Out $R_{\widetilde{G}}$.

Proof. Let $\pi: K \rightarrow \tilde{K}$ be a canonical projection and $s: \tilde{K} \rightarrow K$ be a Borel section, i.e. $\pi \circ s=$ id. Put $E=s(K)$. It can be easily shown that $\mu(E)=\frac{1}{n}$ and the equivalence relation $R_{\widetilde{G}}$ is isomorphic to a reduced equivalence relation 
$R_{G} \mid E$, the isomorphism being given by the section $s$. Hence, $R_{G} \approx R_{\widetilde{G}} \times I_{n}$.

Theorem 3.9. Let $K$ be a connected compact group with a finite center, $\Gamma \subset G$ be countable dense subgroups of $K$, and $\Gamma$ have the T-property. Denote the canonical projection from Aut $R_{G}$ onto Int $R_{G}$ by $\varepsilon$. Then $\varepsilon\left(\left\{r_{t}: t \in K\right\}\right)$ is an open subgroup of Out $R_{G}$ which is topologically isomorphic to $K / Z(G)$.

Proof. Since $Z(G) \subset Z(K)$, then the imbedding of $\tilde{G}=G / Z(G)$ into $K_{1}=$ $K / Z(K)$ is defined, such that the image of $\tilde{G}$ is dense in $K_{1}$. Therefore, in view of 3.7, $\tilde{G}$ is an ICC-group. Now, we can easily show that

$$
\left\{r_{t}: t \in K\right\} \cap \operatorname{Int} R_{G}=\left\{r_{t}: t \in Z(G)\right\} .
$$

Hence, in view of Lemma 3.8, we may believe that $Z(G)=\{e\}$, i.e. $G$ is an ICC-group with respect to $\Gamma$. In view of Proposition $3.2 C_{\Gamma} \subset$ Aut $(M, A)$. According to $2.3, C_{\Gamma}^{M} \cap$ Aut $(M, A)$ is an open subgroup of $\operatorname{Aut}(M, A)$ (see the proof of Theorem 2.3), and so, a subgroup generated algebraically by $\operatorname{Int}(M, A)$ $Z(M, A)$ and $\left\{R_{t}: t \in K\right\}$ is open in $\operatorname{Aut}(M, A)$. Thus, the subgroup generated by Int $R_{G}$ and $\left\{r_{t}: t \in K\right\}$ is an open subgroup of Aut $R_{G}$ (see 2.1). Therefore, $\varepsilon\left(\left\{r_{t}: t \in K\right\}\right)$ is an open subgroup of Out $R_{G}$ which is topologically isomorphic to $K$ (the homeomorphism stems from the continuty continuity of the projection and the $K$-group compactness).

Corollary 3.10. Let the conditions of Theorem 3.9 be fulfilled for $K \supset G \supset \Gamma$. Then the group Out $R_{G}$ is locally connected and its connected component of unity is topologically isomorphic to $K / Z(G)$.

\section{§4. Explicit Description of the Group of Outer Automorphisms for Certain Equivalence Relations}

In this section we proceed with studying the left shift-induced actions of countable dense subgroups of the connected compact group. By using the statement from 3.10, we can determine the groups of outer automorphisms of some dynamical systems. In Section 5 this procedure will be applied to determination of fundamental groups.

Let $K$ be a locally compact group with the Haar measure $\mu, G$ its countable dense subgroup, Aut $K$ a group of automorphisms of the group $K$ (measurable or continuous, which is the same),

$$
\text { Aut }(K, G)=\{\sigma \in \text { Aut } K: \sigma(G)=G\},
$$

and $N_{K}(G)$ the normalizer of $G$ in $K$. 
Theorem 4.1. Let $R_{G}$ be an equivalence relation, generated by left shifts of the group $G$, and $\left\{r_{t}: t \in K\right\}$ the group of right shifts. Assume that $\theta \in$ Aut $R_{G}$ and

$$
\theta r_{t} \theta^{-1}=\delta_{t} r_{\sigma(t)}
$$

for a certain $\sigma \in$ Aut $K$, where $\delta_{t} \in \operatorname{Int} R_{G}, t \in K$. Then, there exist $t_{0} \in K$ and $w \in$ Int $R_{G}$, such that

$$
\begin{aligned}
& t_{0} \sigma(G) t_{0}^{-1}=G \text { and } \theta=w r_{t_{0}} \sigma_{0}, \text { where } \\
& \sigma_{0}=\left(\mathrm{Adt}_{0}\right)^{-1} \sigma \in \operatorname{Aut}(K, G) .
\end{aligned}
$$

Proof. The map $\delta: K \rightarrow \operatorname{Int} R_{G}$,

$$
\delta_{t}=\theta r_{t} \theta^{-1} r \sigma_{(t)}^{-1}
$$

is the cocycle of the action of the group $G$ on the group Int $R_{G}$, i.e.

$$
\delta_{t_{1} t_{2}}=\delta_{t_{1}} r_{\sigma\left(t_{1}\right)} \delta_{t_{2}} r_{\sigma_{\left(t_{1}\right)}}^{-1}, \quad t_{1}, t_{2} \in K
$$

To trivialize this cocycle, let us consider the space $(K \times K, \mu \times \mu)$. Take up the equivalence relation $\bar{R}_{G}=R_{G} \times I$, generated by the action of $G \times K$ on the space $K \times K$ :

$$
l_{(g, t)}\left(k_{1}, k_{2}\right)=\left(g k_{1}, t k_{2}\right), \quad k_{1}, k_{2} \in K, g \in G, t \in K .
$$

Put $\bar{l}_{g}=l_{g} \times \mathrm{id}, \bar{\theta}=\theta \times \mathrm{id}, \bar{\delta}_{t}=\delta_{t} \times \mathrm{id}$,

$$
\bar{r}_{t}=r_{t} \times \mathrm{id}, \quad f_{t}=r_{t} \times r_{t}, \quad f_{t}^{\sigma}=r_{\sigma(t)} \times r_{t}, \quad g \in G, t \in K .
$$

It is evident, that

$$
\bar{\delta}_{t}=\bar{\theta} \bar{r}_{t} \bar{\theta}^{-1} \bar{r}_{\bar{\sigma}(t)}^{-1}, \quad t \in K .
$$

Let us now define the following automorphisms of the space $K \times K$ :

$$
\begin{aligned}
& v\left(k_{1}, k_{2}\right)=\left(\delta_{k_{2}^{-1}}\left(k_{1}\right), k_{2}\right), \quad z\left(k_{1}, k_{2}\right)=\left(k_{1}, k_{2} k_{1}^{-1}\right), \\
& z_{\sigma}\left(k_{1}, k_{2}\right)=\left(k_{1}, k_{2} \sigma^{-1}\left(k_{1}\right)^{-1}\right), \\
& \bar{\theta}_{1}=v^{-1} \bar{\theta}, \quad \bar{\theta}_{2}=z_{\sigma} \bar{\theta}_{1} z^{-1} .
\end{aligned}
$$

Since $\delta_{t} \in \operatorname{Int} R_{G}$, then $\bar{\delta}_{t} \in \operatorname{Int} \bar{R}_{G}$. It is evident, that $z, z_{\sigma} \in \operatorname{Int} R_{G}$. Therefore, $\bar{\theta}_{1}, \bar{\theta}_{2} \in$ Aut $R_{G}$.

Lemma 4.2 (a) $\bar{\delta}_{t}=v f_{t}^{\sigma} v^{-1} f_{t-1}^{\sigma}$;

(b) $\bar{\theta}_{1} f_{t}=f_{t}^{\sigma} \bar{\theta}_{1}$;

(c) $z f_{t} z^{-1}=\bar{r}_{t}, z_{\sigma} f_{t}^{\sigma} z_{\sigma}^{-1}=\bar{r}_{\sigma(t)}$;

(d) $\bar{\theta}_{2} \bar{r}_{t}=\bar{r}_{\sigma(t)} \bar{\theta}_{2}, t \in K$. 
Proof. The equality (a) follows from the definition of the automorphisms $v$ and $f_{t}^{\sigma}$ and identity (4.1). In view of (4.2) and (a) $v f_{t}^{\sigma} v^{-1} f_{t^{-1}}^{\sigma}=\theta \bar{r}_{t} \theta^{-1} \bar{r}_{\sigma(t)}^{-1}$, whence

$$
v f_{t}^{\sigma} v^{-1}=\left(\bar{\theta} \bar{r}_{s} \bar{\theta}^{-1}\right)\left(\mathrm{id} \times r_{t}\right)=\bar{\theta} f_{t} \bar{\theta}^{-1} .
$$

Then $v^{-1} \bar{\theta} f_{t}=f_{t}^{\sigma} v^{-1} \bar{\theta}$, and thus the equality (b) is proved. The statement (c) can be easily verified, and (d) follows from (b) a and (c).

Lemma 4.3. Let the Borel map $a: K \rightarrow K$ be such that $a\left(k t^{-1}\right)=a(k) \sigma(t)^{-1}$ for almost all (a.a.) $k, t \in K$. Then $a(k)=t_{0} \sigma(k)$ for certain $t_{0} \in K$ and for a.a. $k \in K$.

Proof. Let us consider the Borel map $\varphi: K \rightarrow K$

$$
\varphi(k)=a(k) \sigma(k)^{-1} \text {. }
$$

Then $\varphi\left(k t^{-1}\right)=\varphi(k)$ for a.a. $k, t \in K$. Based on this fact, one can easily find that $\varphi(k)=t_{0}$ for certain $t_{0} \in K$ and for a.a. $k \in K$, i.e. $a(k)=t_{0} \sigma(k)$.

Proof of Theorem 4.1 (continuation). Let us consider the Borel maps $a$ and $b$ from $K \times K$ to $K$, such that

$$
\bar{\theta}_{2}\left(k_{1}, k_{2}\right)=\left(a\left(k_{1}, k_{2}\right), b\left(k_{1}, k_{2}\right)\right) \text {. }
$$

Then $\left(\bar{\theta}_{2} \bar{r}_{t}\right)\left(k_{1}, k_{2}\right)=\left(a\left(k_{1} t^{-1}, k_{2}\right), b\left(k_{1} t^{-1}, k_{2}\right)\right)$,

$$
\left(\bar{r}_{\sigma(t)} \bar{\theta}_{2}\right)\left(k_{1}, k_{2}\right)=\left(a\left(k_{1}, k_{2}\right) \sigma(t)^{-1}, b\left(k_{1}, k_{2}\right)\right) .
$$

Using Lemma 4.2 (d), we have

$$
\begin{aligned}
& a\left(k_{1} t^{-1}, k_{2}\right)=a\left(k_{1}, k_{2}\right) \sigma(t)^{-1}, \\
& b\left(k_{1} t^{-1}, k_{2}\right)=b\left(k_{1}, k_{2}\right)
\end{aligned}
$$

for all $t \in K$ and a.a. $k_{1}, k_{2} \in K$. Using Lemma 4.3 and the transitivity of the action $r$, we can obtain

$$
a\left(k_{1}, k_{2}\right)=\alpha\left(k_{2}\right) \sigma\left(k_{1}\right), \quad b\left(k_{1}, k_{2}\right)=\beta\left(k_{2}\right)
$$

for a.a. $k_{2} \in K$ at a.a. $k_{1} \in K$. It is easy to see that the resulting maps $\alpha$ and $\beta$ are Borel's. But then, by the Fubini theorem, we have

$$
a\left(k_{1}, k_{2}\right)=\alpha\left(k_{2}\right) \sigma\left(k_{1}\right), \quad b\left(k_{1}, k_{2}\right)=\beta\left(k_{2}\right)
$$

for a.a. $\left(k_{1}, k_{2}\right) \in K_{1} \times K_{2}$. Hence,

$$
\bar{\theta}_{2}\left(k_{1}, k_{2}\right)=\left(\alpha\left(k_{2}\right) \sigma\left(k_{1}\right), \beta\left(k_{2}\right)\right)
$$


for a.a. $k_{1}, k_{2} \in K$. Let us show, that for a.a. $k_{2} \in K$,

$$
\alpha\left(k_{2}\right) \sigma(G) \alpha\left(k_{2}\right)^{-1}=G
$$

Since $\bar{\theta}_{2} \in$ Aut $\bar{R}_{G}$, then

$$
\left(\bar{\theta}_{2}\left(g k_{1}, k_{2}\right), \bar{\theta}_{2}\left(k_{1}, k_{2}\right)\right) \in \bar{R}_{G}=R_{G} \times I
$$

for all $g \in G$ at a.a. $k_{1}, k_{2} \in K$. Hence, in view of (4.3),

$$
\begin{aligned}
& \left(\alpha\left(k_{2}\right) \sigma\left(g k_{1}\right), \alpha\left(k_{2}\right) \sigma\left(k_{1}\right)\right) \in R_{G}, \text { i.e. } \\
& \alpha\left(k_{2}\right) \sigma\left(g k_{1}\right)\left(\alpha\left(k_{2}\right) \sigma\left(k_{1}\right)\right)^{-1}=\left(\operatorname{Ad} \alpha\left(k_{2}\right) \sigma\right)(g) \in G
\end{aligned}
$$

for all $g \in G$ and a.a. $k_{2} \in K$. Similarly we can verify that $\left(\operatorname{Ad} \alpha\left(k_{2}\right) \sigma\right)^{-1}(g) \in G$. Thus, equality (4.4) is proved. Then, $\bar{\theta}_{2}=z_{\sigma} \bar{\theta}_{1} z^{-1}=z_{\sigma} \bar{\theta}_{1} z^{-1} \bar{\theta}_{1}^{-1} v^{-1} \bar{\theta}=u \bar{\theta}$, where $u=z_{\sigma} \bar{\theta}_{1} z^{-1} \bar{\theta}_{1}^{-1} v^{-1} \in \operatorname{Int} \bar{R}_{G}$. Now from equality (4.3) we have $\left(\alpha\left(k_{2}\right) \sigma\left(k_{1}\right), \beta\left(k_{2}\right)\right)$ $=\left((u \bar{\theta})\left(k_{1}, k_{2}\right)\right)=u\left(\theta\left(k_{1}\right), k_{2}\right)$. But $u \in \operatorname{Int} \bar{R}_{G}$ and therefore

$$
\left(\alpha\left(k_{2}\right) \sigma\left(k_{1}\right), \theta\left(k_{1}\right)\right) \in R_{G}
$$

for a.a. $k_{1}, k_{2} \in K$. Let us fix the element $k_{2}$ which belongs to the set of complete measure, and put

$$
t_{0}=\alpha\left(k_{2}\right)^{-1}, \quad \sigma_{0}(k)=t_{0}^{-1} \sigma(k) t_{0}, \quad \theta_{1}=r_{t_{0}} \sigma_{0} .
$$

Because of equality (4.4), $\theta_{1} \in$ Aut $R_{G}$. Since

$$
\theta_{1}(k)=\sigma_{0}(k) t_{0}^{-1}=t_{0}^{-1} \sigma(k)=\alpha\left(k_{2}\right) \sigma(k),
$$

then $\left(\theta_{1}(k), \theta(k)\right) \in R_{G}$ for a.a. $k \in K$. Hence, $w_{1}=\theta^{-1} \theta_{1} \in \operatorname{Int} R_{G}$. So,

$$
\theta=\theta_{1} w_{1}^{-1}=w \theta_{1}=w r_{t_{0}} \sigma_{0},
$$

where $w=\theta_{1} w_{1}^{-1} \theta_{1}^{-1} \in \operatorname{Int} R_{G}$. The theorem is proved.

Now we can find all automorphisms of the equivalence relation $R_{G}$.

Theorem 4.4. Let $K$ be a connected compact group with the trivial center, and $G$ its countable dense subgroup, which contains the dense subgroup in $K$, such that it has the T-property. Let us consider the equivalence relation $R_{G}$, generated by left shifts of the group $G$, and let $\theta \in$ Aut $R_{G}$. Then there exist $k \in K$, $\sigma \in \operatorname{Aut}(K, G)$ and $w \in \operatorname{Int} R_{G}$ such that $\theta=w r_{k} \sigma$.

Proof. By Theorem 3.9, the group $\varepsilon\left(\left\{r_{t}: t \in K\right\}\right)$ is a connected component of unity and thus a normal subgroup of Out $R_{G}$. Then $\varepsilon(\theta)$ defines an automorphism of the group $\varepsilon\left(\left\{r_{t}: t \in K\right\}\right)$, which is isomorphic to $K$. Hence, 


$$
\varepsilon(\theta) \varepsilon\left(r_{t}\right) \varepsilon(\theta)^{-1}=\varepsilon\left(r_{\sigma_{1}(t)}\right)
$$

for some $\sigma_{1} \in$ Aut $K$ and all $t \in K$. Therefore,

$$
\theta r_{t} \theta^{-1} r_{\sigma_{1}(t)}^{-1}=\delta_{t} \in \operatorname{Int} R_{G}, \quad t \in K .
$$

By Theorem 4.1, there exist $k \in K$ and $w \in \operatorname{Int} R_{G}$ for which

$$
\sigma=\left(\operatorname{Ad~} k^{-1}\right) \sigma_{1} \in \operatorname{Aut}(K, G) \text { and } \theta=w r_{k} \sigma \text {. }
$$

Corollary 4.5. Any automorphism from Aut $R_{G}$, with the accuracy to the inner automorphism and the automorphism of $G$, belongs to the centralizer of the action of $G$.

To describe the structure of the group Out $R_{G}$, we shall use the following statement.

Lemma 4.6. Let $K$ be a connected compact group, $G$ its countable dense subgroup, $k \in K$ and $\sigma \in \operatorname{Aut}(K, G)$. Then the automorphism $r_{k} \sigma \in \operatorname{Int} R_{G}$ if and only if $k \in G$ and $\sigma=\operatorname{Ad~} k^{-1}$.

Proof. Let us assume that $r_{k} \sigma \in \operatorname{Int} R_{G}$. Then, for a certain $g \in G$, the set

$$
E_{g}=\left\{s \in K:\left(r_{k} \sigma\right)(s)=g s\right\}
$$

has positive measure. Consider the closed subgroup $H_{g}$, which is generated by the set $\left\{s_{2}^{-1} s_{1}: s_{1}, s_{2} \in E_{g}\right\}$. Certainly, $\mu\left(H_{g}\right)>0$; since the group $K$ is connected, $H_{g}=K$. Next, if $s_{1}, s_{2} \in E_{g}$, then $\sigma\left(s_{1}\right)=g s_{1} k$ and $\sigma\left(s_{2}^{-1}\right)=k^{-1} s_{2}^{-1} g^{-1}$. Then $\sigma\left(s_{2}^{-1} s_{1}\right)=\sigma\left(s_{2}^{-1}\right) \sigma\left(s_{1}\right)=k^{-1} s_{2}^{-1} s_{1} k$. Consequently, $\sigma=\mathrm{Ad} k^{-1}$ and $\left(r_{k} \sigma\right)(s)=$ $k^{-1} s=g s, s \in E_{g}$, i.e. $k \in G$.

Corollary 4.7. Let $G$ and $K$ satisfy all the conditions of Theorem 4.4. Let us identify the group $K$ with its image in Out $R_{G}$ and imbed $G$ as a normal subgroup into Aut $(K, G)$ (it is possible because $Z(G)=Z(K)=\{e\}$ ). Then,

Out $R_{G} / K \approx \operatorname{Aut}(K, G) / G$.

Proof. Let $\theta \in$ Aut $R_{G}$ and [ $\left.\theta\right]$ be its image in the group Out $R_{G} / K$. Then let us define map

$$
f: \text { Out } R_{G} / K \rightarrow \operatorname{Aut}(K, G) / G, f([\theta])=[\sigma],
$$

where $\theta=w r_{k} \sigma$ (see Theorem 4.4) and [ $\left.\sigma\right]$ is the image of $\sigma$ in $\operatorname{Aut}(K, G) / G$. Correctness of the definition follows from Lemma 4.6. Then, it is easy to verify that $f$ is an isomorphism. 
Let us consider an important particular case.

Corollary 4.8. Let us assume that $N_{K}(G)=G$, and the group $\operatorname{Aut}(K, G)$ is the semidirect product of $F$ and $\operatorname{Int}(K, G) \approx N_{K}(G)$. Then any automorphism $\theta \in$ Aut $R_{G}$ has the unique decomposition $\theta=w r_{k} \sigma$, where $k \in K$ and $\sigma \in F$. Thus, Out $R_{G} \approx F$ (S) $K$.

Proof. By Lemma 4.6,

$$
\{\varepsilon(\sigma): \sigma \in F\} \cap\left\{\varepsilon\left(r_{k}\right): k \in K\right\}=\{e\} .
$$

Besides, $\sigma r_{k} \sigma^{-1}=r_{\sigma(k)}, k \in K$. Therefore $F\left(K\right.$ is imbedded into Out $R_{G}$. Then, since Aut $(K, G)=F\left(N_{K}(G)\right.$, then by Theorem 4.4, for $\theta \in$ Aut $R_{G}$ we have $\theta=w_{1} r_{k} l_{t} \sigma$, where $t \in N_{K}(G)$ and $\sigma \in F$. Since $N_{K}(G)=G$, then $l_{t} \in \operatorname{Int} R_{G}$, i.e. $\theta=w r_{k} \sigma$.

Theorem 4.9. Let $K$ be a connected compact group with a finite center, $G$ its countable dense subgroup, containing a dense subgroup in $K$ with the T-property. Assume that the following conditions are fulfilled:

(a) $G$ is a subgroup with a finite index in $N_{K}(G)$ and $Z(G)=Z(K)$.

(b) The group $K / Z(K)$ has a finite group of outer automorphisms.

Then, Out $R_{G}$ is a locally connected compact group and its connected component of unity is topologically isomorphic to $K / Z(K)$.

Proof. Put $\tilde{K}=K / Z(G), \tilde{G}=G / Z(G)$ and consider the action of the group $\tilde{G}$ on $\tilde{K}$. In view of Lemma 3.8, we see that Out $R_{G} \approx$ Out $R_{\tilde{G}}$. According to Corollary 4.7 ,

$$
\text { Out } R_{\tilde{G}} / \tilde{K} \approx \operatorname{Aut}(\tilde{K}, \tilde{G}) / \tilde{G} \text {. }
$$

Since $Z(\tilde{G})=\{e\}$, then it follows from the condition (a) that $[\operatorname{Int}(\tilde{K}, \tilde{G}): \tilde{G}]=$ $\left[N_{K}(\tilde{G}): \tilde{G}\right]<\infty$. Now, on the basis of the condition (b), we may state that the group Aut $(\tilde{K}, \tilde{G}) / \widetilde{G}$ is finite, i.e. $\tilde{K}$ is a subgroup of a finite index in Out $R_{\tilde{G}}$. Therefore, Out $R_{\widetilde{G}}$ is a compact group.

Example 4.10. Let $K=\mathrm{SO}(n, \boldsymbol{R}), G=\mathrm{SO}(n, \boldsymbol{Q}), \boldsymbol{\Gamma}=\mathrm{SO}(n, \boldsymbol{Z}[1 / p])$, where $p$ is a prime number such that $p \equiv 1(\bmod 4)$, and $Z[1 / p]$ be a subring in $\boldsymbol{Q}$ generated by $\boldsymbol{Z}$ and $1 / p, n \geqslant 5$. We can show that $K, G$ and $\Gamma$ satisfy the conditions of Theorem 4.9. The group $\Gamma$ is dense in $K$ and has the T-property in accordance with [6, Proposition 5]. It is evident that $K$ is connected and has a finite center. We can easily prove that $N_{K}(G)=G$. Then if $n=2 k+1$, then $Z(K)=\{1\}$, but if $n=2 k$, then $Z(K)=Z(G)=\{ \pm 1\}, K / Z(K)=\operatorname{PSO}(n, \boldsymbol{R})$. The 
group $K / Z(K)$ has a finite group of outer automorphisms in accordance with the results in [19] (see Ch. IV, $\S \S 4,5,7$ ).

Corollary 4.11. Let $K=\operatorname{SO}(n, \boldsymbol{R}), G=\operatorname{SO}(n, \boldsymbol{Q}), n \geqslant s, n \neq 8$. Then,

$$
\text { Out } R_{G} \approx \begin{cases}\mathrm{SO}(n, \boldsymbol{R}), & n=2 k+1 \\ \mathrm{PO}(n, \boldsymbol{R}), & n=2 k\end{cases}
$$

Proof. If $n$ is odd, then $Z(K)=\{1\}$ and all automorphisms of the group $K$ are inner (see [19, Ch. IV, $\S 4$ and 5]). Since $N_{K}(G)=G$, then, by Corollary 4.7 , Out $R_{G} \approx K$. Let now $n$ be even. Then, the group $K / Z(K)=\operatorname{PSO}(n, \mathbb{R})$ has a single outer automorphism resulting from factorization of the automor-

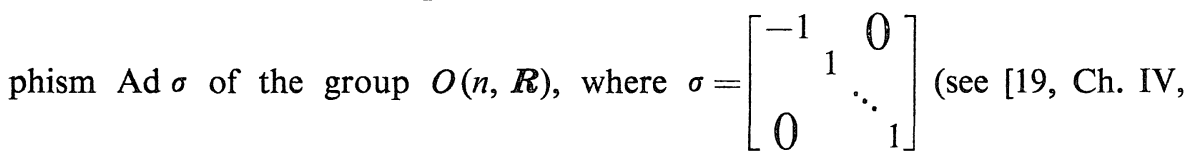
$\S \S 4,5,7])$. By applying Corollary 4.8 , we obtain

$$
\text { Out } R_{G} \approx \mathbb{Z} / 2 \mathbb{Z} \text { S PSO }(n, \boldsymbol{R}) \approx \mathrm{PO}(n, \boldsymbol{R})
$$

\section{§5. The Equivalence Relations with a Unit Fundamental Group}

We can now define the fundamental group of the dynamical system generated by the dense imbedding of countable group into connected compact one.

Consider the action $l$ of the group $G \times \mathbb{Z}$ on the space with measure $(K \times \mathbb{Z}, \mu \times \delta)$, where $\mu \times \delta$ is the Haar measure on

$$
K \times \mathbb{Z}: l_{(g, n)}(k, m)=(g k, m+n) .
$$

Put $\tilde{A}=L^{\infty}(K \times \mathbb{Z}, \mu \times \delta), \tilde{M}=W^{*}(\tilde{A}, l, G \times \boldsymbol{Z})$. Then $\tilde{M}=M \otimes B$, where

$$
B=B\left(l^{2}(\mathbb{Z})\right), \quad M=W^{*}(A, l, G), \quad A=L^{\infty}(K, \mu) .
$$

Lemma 5.1. Let $K$ be a connected compact group with a trivial center, $\Gamma \subset G$ be dense countable subgroups of $K$. Put

$$
\tilde{C}_{\Gamma}=\left\{\tilde{\theta} \in \text { Aut } \tilde{M}: \tilde{\theta}\left(\lambda_{\gamma} \otimes 1\right)=\lambda_{\gamma} \otimes 1, r \in \Gamma\right\} .
$$

If $\tilde{\theta} \in \tilde{C}_{\Gamma}$, then $\tilde{\theta}=\left(\theta_{c} R_{t}\right) \otimes \operatorname{Ad} w$, where $c \in Z^{1}(G, U(A)), t \in K, w \in U(B)$.

Proof. According to 3.7, $G$ is a ICC-group with respect to $\Gamma$ and so, $\left\{\lambda_{\gamma} \otimes 1: r \in \Gamma\right\}^{\prime} \cap \tilde{M}=1 \otimes B$ (see the equality (1.2) in the proof of Theorem 1.7). Then $\tilde{\theta}(1 \otimes B)=1 \otimes B$ and $\tilde{\theta}(M \otimes 1)=M \otimes 1$. Hence, $\tilde{\theta}=\theta \otimes$ Ad $w$, where $w \in U(B)$ and $\theta\left(\lambda_{\gamma}\right)=\lambda_{\gamma}, \gamma \in \Gamma$. According to 3.3, $\theta=\theta_{c} R_{t}$. 
Proposition 5.2. Let the conditions of Lemma 5.1 be fulfilled for $K \supset G \supset \Gamma$ and the group $\Gamma$ have the T-property. Consider the equivalence relation $\widetilde{R}_{G}=$ $R_{G} \times I_{\infty}$ and denote the canonical projection from Aut $\widetilde{R}_{G}$ on Out $\widetilde{R}_{G}$ by $\varepsilon$. Then

(a) Int $\widetilde{R}_{G}$ is a closed subgroup of Aut $\widetilde{R}_{G}$, and thus Out $\widetilde{R}_{G}$ is a Polish group.

(b) $\varepsilon\left(\left\{r_{t} \times \mathrm{id}: t \in K\right\}\right)$ is an open subgroup of Out $\widetilde{R}_{G}$ which is topologically isomorphic to $K$.

Proof. According to [17, 2.11 (b) and 3.6], the factor $\tilde{M}$ is full. Therefore, Int $\widetilde{R}_{G}$ is a closed subgroup of Aut $\widetilde{R}_{G}$. Denote the subgroup of Aut $\tilde{M}$, generated algebraically by Int $\tilde{M}$ and $\tilde{C}_{\Gamma}$, by $C_{\Gamma}^{\widetilde{M}}$ (see Lemma 5.1). From the arguments given when proving Theorem 1.1 [4], $C_{\Gamma}^{\tilde{\pi}}$ is an open subgroup of Aut $\tilde{M}$. The statement (b) can be proved in the same way as 3.9 (with taking into account 5.2 (a) and Lemma 5.1).

Proposition 5.3. Let $G$ be a countable dense subgroup of the compact group $K, \tilde{R}_{G}=R_{G} \times I_{\infty}$. Assume that $\theta \in$ Aut $\tilde{R}_{G}$ and $\theta\left(r_{t} \times \mathrm{id}\right) \theta^{-1}=\delta_{t}\left(r_{\sigma(t)} \times\right.$ id $)$ for a certain $\sigma \in$ Aut $K$, where $\delta_{t} \in \operatorname{Int} \widetilde{R}_{G}, t \in K$. Then, there exist $t_{0} \in K$ and $w \in$ Int $\widetilde{R}_{G}$, such that $t_{0}^{-1} \sigma(G) t_{0}=G$ and $\theta=w\left(r_{t_{0}} \sigma_{0} \times \mathrm{id}\right)$, where $\sigma_{0}=\left(\operatorname{Ad} t_{0}^{-1}\right) \sigma \in$ $\operatorname{Aut}(K, G)$. Thus, the automorphism $\theta$ preserves the measure $\mu \times \delta$.

The proof is carried out in the same way as that of Theorem 4.1.

Theorem 5.4. Let $K$ be a connected compact group with a finite center, $G$ its countable dense subgroup containing a subgroup, which is dense in $K$ and has the property $T$. Consider the equivalence relation $R_{G}$ generated by left shifts of $G$, and let $\widetilde{R}_{G}=R_{G} \times I_{\infty}$. Then all automorphisms from Aut $\widetilde{R}_{G}$ preserve measure, and thus, the fundamental group of the equivalence relation $R_{G}$ is trivial, i.e. $F\left(R_{G}\right)=\{1\}$.

Proof. In view of Lemma 3.8, we may believe that $Z(G)=Z(K)=\{e\}$. Then, by Proposition 5.2 (b), the group

$$
\varepsilon\left(\left\{r_{t} \times \mathrm{id}: t \in K\right\}\right)
$$

is a connected component of unit, and therefore it is the normal subgroup of Out $\widetilde{R}_{G}$. Thus, if $\theta \in$ Aut $\widetilde{R}_{G}$, then there exists $\sigma \in$ Aut $K$, for which

$$
\varepsilon(\theta) \varepsilon\left(r_{t} \times \mathrm{id}\right) \varepsilon(\theta)^{-1}=\varepsilon\left(r_{\sigma(t)} \times \mathrm{id}\right), \quad t \in K .
$$

Hence, $\theta\left(r_{t} \times \mathrm{id}\right) \theta^{-1}\left(r_{\sigma(t)}^{-1} \times \mathrm{id}\right)=\delta_{t} \in \operatorname{Int} \widetilde{R}_{G}$, and, according to Proposition 5.3, the automorphism $\theta$ preserves measure. 
Corollary 5.5. Let $P$ and $Q$ be measurable subsets of $K$ of the positive measure. The reduced equivalence relations $R \mid P$ and $R \mid Q$ are isomorphic if and only if $\mu(P)=\mu(Q)$ (see 1.1 and 1.2).

Corollary 5.6. The equivalence relations $R_{G}$ and $R_{G} \times I_{n}$ are not isomorphic $(n \geqslant 2)$.

Corollary 5.7. Let for $G \subset K$ all the conditions of Theorem 4.9 be fulfilled and $\widetilde{R}_{G}=R_{G} \times I_{\infty}$. Then the group Out $\widetilde{R}_{G}$ is compact.

Proof. According to Theorem 5.4,

Aut $\widetilde{R}_{G}=$ Aut $_{0} \widetilde{R}_{G}$. Therefore, Out $\widetilde{R}_{G} \approx$ Out $R_{G}$.

Corollary 5.8. The equivalence relations given in $\$ 4$ have trivial fundamental groups (see Example 4.10).

\section{Supplement A. On Orbit-Non-Equivalent Ergodic Actions of Arithmetic Groups}

In this Supplement the results of $\S 3$ and the methods developed in $[5,20]$ are used to construct orbit-non-equivalent ergodic actions. It is shown that arithmetic groups with the property $T$ have a continuum of stably orbit-nonequivalent free ergodic actions with a finite invariant measure (Theorem A.8).

Definition A.1. The actions $\alpha_{1}$ and $\alpha_{2}$ of the countable groups $\Gamma_{1}$ and $\Gamma_{2}$ are called stably orbit-non-equivalent, if

$$
R_{\Gamma_{1}} \times I \approx R_{\Gamma_{2}} \times I
$$

where $R_{\Gamma_{i}}$ is the equivalence relation generated by the action $\alpha_{i}(i=1,2)$ and $I$ the transitive equivalence relation generated by translation of a circle.

In view of [10, Theorem 3] and [21, 4.6 and 4.10], the ergodic actions $\alpha_{1}$ and $\alpha_{2}$ are stably orbit-equivalent if and only if

$$
R_{\Gamma_{1}} \times I_{\infty} \approx R_{\Gamma_{2}} \times I_{\infty}
$$

Proposition A.2. Let $K$ be a compact group and $\Gamma$ its countable dense subgroup with the property $T$ and a finite center and such that $\Gamma / Z(\Gamma)$ is an ICC-group. Consider the factor $M$ constructed by the action of $\Gamma$ by means of left shifts on $(K, \mu)$ and put $\tilde{M}=M \otimes B$, where $B$ is a type $I_{\infty}$ factor. Then the group Out $\tilde{M}$ contains an open subgroup topologically isomorphic to $K / Z(\Gamma)$. 
Proof. In view of Lemma $3.8, \tilde{M} \approx M_{1} \otimes B$, where $M_{1}$ is a factor constructed by the action $\Gamma / Z(\Gamma)$ on the space $K / Z(\Gamma)$. Therefore, we may believe that $Z(\Gamma)=\{e\}$ and $\Gamma$ is an ICC-group. Now our statement follows from the arguments used to prove Lemma 5.1 and Proposition 5.2.

Proposition A.3. Let $\Gamma$ be a group with the property $T$ and a finite center such that $\Gamma / Z(\Gamma)$ is an ICC-group. Assume $\Gamma$ to be densely imbedded into the compact groups $K_{1}$ and $K_{2}$ and $l_{i}$ to be its action by left shifts on the space $\left(K_{i}, \mu_{i}\right), i=1,2$. If the actions $l_{1}$ and $l_{2}$ are stably orbit-equivalent, then the groups $K_{1} / Z(\Gamma)$ and $K_{2} / Z(\Gamma)$ contain topologically isomorphic open subgroups.

Proof. Let the actions $l_{1}$ and $l_{2}$ be stably orbit-equivalent,

$$
M_{i}=W^{*}\left(L^{\infty}\left(K_{i}, \mu_{i}\right), l_{i}, \Gamma\right) \quad \text { and } \quad \tilde{M}_{i}=M_{i} \otimes B .
$$

Then, $\tilde{M}_{1} \approx \tilde{M}_{2}$. By Proposition A.2, the groups $K_{1} / Z(\Gamma)$ and $K_{2} / Z(\Gamma)$ may be regarded as open subgroups of Out $\tilde{M}_{1}$. Hence, $\left(K_{1} / Z(\Gamma)\right) \cap\left(K_{2} / Z(\Gamma)\right)$ is an open subgroup in $K_{i} / Z(\Gamma), i=1,2$.

For the case of lattices in semisimple Lie groups we arrive at the following statement.

Theorem A.4. Let $H$ be a connected semisimple Lie group with a finite center, without compact factors and factors of $\boldsymbol{R}$-rank 1 , and $\Gamma$ be a lattice in $H$. If $\boldsymbol{\Gamma}$ is densely imbedded into the compact groups $K_{1}$ and $K_{2}$ and its actions by left shifts are stably orbit-equivalent, then the groups $K_{1} / Z(\Gamma)$ and $K_{2} / Z(\Gamma)$ contain topologically isomorphic open subgroups.

Theorem A.4 shows that the nonequivalent actions of the group $\Gamma$ can be constructed by merely imbedding it densely into various compact groups. Such imbeddings may be constructed for a broad class of arithmetic groups.

Let $G \subset \operatorname{SL}(n, \boldsymbol{C})$ be a semisimple algebraic group defined over the field $\boldsymbol{Q}$ (see [13, 22-24]). Put $G_{\boldsymbol{Q}}=G \cap \operatorname{SL}(n, \boldsymbol{Q})$ and $G_{\boldsymbol{Z}}=G \cap \operatorname{SL}(n, \boldsymbol{Z})$. By an arithmetic group we shall mean a subgroup $\Gamma$ in $G_{Q}$ commensurable with the group $G_{\boldsymbol{Z}}$ (i.e. $\Gamma \cap G_{\boldsymbol{Z}}$ has a finite index both in $\Gamma$ and in $G_{\boldsymbol{Z}}$ ).

Let us construct now orbit-non-equivalent actions of the arithmetic group $\Gamma$, assuming that $\Gamma=G_{\boldsymbol{Z}}$. Consider the field of $p$-adic numbers $\boldsymbol{Q}_{\boldsymbol{p}}$ for a prime number $p$ and denote by $G_{\boldsymbol{Q}_{p}}$ the group of the $\boldsymbol{Q}_{p}$-points of the group $G$ (see [22-24]). The group $G_{Q_{p}}$ provided with $p$-adic topology is a locally compact totally disconnected group. Put $G_{Z_{p}}=G_{\boldsymbol{Q}_{p}} \cap \operatorname{SL}\left(n, \boldsymbol{Z}_{p}\right)$, where $\boldsymbol{Z}_{p}$ is a ring of $p$-adic integers. Then, $G_{Z_{p}}$ is an open compact subgroup $G_{\boldsymbol{Q}_{\boldsymbol{p}}}$. Now, for 
the subset $J$ in the set of prime numbers, put $K(J)=\prod_{p \in J} K(p)$, where $K(p)=G_{\boldsymbol{Z}_{p}}$. Since the group $G_{Z}$ is imbedded into $G_{Z p}$ for all $p$, then there arises the diagonal imbedding $\Gamma=G_{\boldsymbol{Z}}$ into $K(J)$. The following statement is a corollary of the strong approximation theorem for the algebraic groups [23, § 4].

Proposition A.5. Let $G$ be a connected simply connected semisimple algebraic group, defined and almost simple over $\boldsymbol{Q}$. Assume that the group $G_{\mathbb{R}}$ of its real points is not compact. Then the group $G_{Z}$ is densely imbedded into $K(J)$.

Lemma A.6. If $J_{1} \neq J_{2}$, then the groups $K\left(J_{1}\right)$ and $K\left(J_{2}\right)$ do not contain topologically isomorphic open subgroups. The similar statement is true for the groups $K\left(J_{i}\right) / F_{i}$, where $F_{i}$ is the finite normal subgroup of $K\left(J_{i}\right), i=1,2$.

Proof. Any open subgroup of $K(J)$ contains the open subgroup $L$ of the following form:

$$
L=\left\{\prod_{p \in S} K_{m_{p}}(p)\right\} \times\left\{\prod_{p \in J \backslash S} K(p)\right\} ，
$$

where $S$ is the finite subset $J$ and $K_{m_{p}}(p)$ the open subgroup $K(p)$. In the decomposition (A.1) every multiplier is a closed subgroup $\operatorname{SL}\left(n, \mathbb{Z}_{p}\right)$ for a certain $p$ and thus a $p$-adic Lie group [25]. Now the statement of the lemma is easy to derive from the following results of the theory of $p$-adic Lie groups [25, Ch. III]: (a) if $\varphi: K \rightarrow N$ is a homomorphism of the $p$-adic group into a $q$-adic group $(p \neq q)$, then $\varphi(K)$ is a finite group; (b) in any $p$-adic Lie group there exists an open subgroup which does not contain finite subgroups.

Proposition A.7. Let $G$ satisfy all the conditions of Proposition A.5 and besides the group of its real points $G_{\boldsymbol{R}}$ have no compact factors and factors of the $\boldsymbol{R}$-rank 1. Let $l_{J}$ be a ergodic action of the group $\Gamma=G_{Z}$ on the space $K(J)$ generated by left shifts (see Proposition A.5). Then, if $J_{1} \neq J_{2}$, then the actions $l_{J_{1}}$ and $l_{J_{2}}$ are stably orbit-non-equivalent. Hence, the group $\Gamma$ has a continuum of stably orbit-non-equivalent free ergodic actions preserving finite measure.

Proof. Since $G$ is a simply connected semisimple algebraic group, then $G_{\boldsymbol{R}}$ is a connected semisimple Lie group with a finite center. According to [24], $\Gamma$ is a lattice in $G_{\boldsymbol{R}}$. Now our statement follows directly from A.4 and A.6.

Theorem A.8. Let $\Gamma$ be an arithmetic subgroup of the connected semisimple algebraic group $G$, defined and almost simple over $\mathbf{Q}$. Assume that the group $G_{\mathbb{R}}^{0}$ has no compact factors and factors of the $\boldsymbol{R}$-rank 1 . Then, $\Gamma$ has a con- 
tinuum of stably orbit-non-equivalent free ergodic actions with finite invariant measure, and the factors of type $I I_{1}$, constructed by these actions, are pairwise stably non-isomorphic.

Proof. Assume first $G$ to be simply connected, and let $\Gamma_{0}=\Gamma \cap G_{Z}$. Then, $\left[\Gamma: \Gamma_{0}\right]<\infty$ and $\left[G_{Z}: \Gamma_{0}\right]<\infty$. Any action of the group $\Gamma_{0}$ and the action of $\Gamma$ induced by it are stably orbit-equivalent (see [26]). Therefore, the statement of the theorem is sufficient to prove for the group $\Gamma_{0}$. Let $K_{0}(J)=\bar{\Gamma}_{0}$ be the closure of $\Gamma_{0}$ in the group $K(J)=\bar{G}_{Z}$ (see Proposition A.5). Then $K_{0}(J)$ is the open subgroup $K(J)$. Hence, the actions $G_{Z}$ on $K(J)$ and $\Gamma_{0}$ on $K_{0}(J)$ are stably orbit-equivalent, and we may apply Proposition A.7. Let now $G$ be not simply-connected. Then, there exists the simple-connected almost $\boldsymbol{Q}$-simple algebraic $\boldsymbol{Q}$-group $\tilde{G}$ and the covering $\varphi: \tilde{G} \rightarrow G$ defined over $\boldsymbol{Q}$, where $\operatorname{ker} \varphi$ is finite. Consider in $\tilde{G}$ the arithmetic subgroup $\tilde{\Gamma}$. According to [13, Theorem 10.20], $\varphi(\tilde{\Gamma})$ is the arithmetic subgroup in $G$. For $\tilde{\Gamma}$ the statement of the theorem has been proved. In view of Lemma 3.8 and the commensurability of the groups $\Gamma$ and $\varphi(\tilde{\Gamma})$, the theorem is true also for the group $\Gamma$.

By applying the G.A. Margulis' arithmeticity theorem (see [22, Theorem 9.7.1 and $\S 10]$ ), we obtain

Corollary A.9. Let $\Gamma$ be an irreducible nonuniform lattice in a connected semisimple Lie group with a finite center, without compact factors and factors of the $\boldsymbol{R}$-rank 1. Then, the group $\Gamma$ has a continuum of stably orbit-non-equivalent free ergodic actions with finite invariant measure.

By using this result and the construction of the induced action [26], we arrive at a similar also for semisimple Lie groups with the property $T$ (see also [22]).

Corollary A.10. Let $H$ be a connected noncompact semisimple Lie group with a finite center and without factors of the $\boldsymbol{R}$-rank 1 . Then, $H$ has a continuum of orbit-non-equivalent free ergodic actions with finite invariant measure.

\section{Supplement B. The Fundamental Group for Ergodic Actions of Semisimple Lie Groups and Their Lattices}

Let $H$ be a continuous locally compact separable unimodular group, acting freely and ergodically proper on the Lebesgue space $(X, \mu)$ with invariant (finite or $\sigma$-finite) measure $\mu$. Consider the corresponding ergodic equivalence relation $R_{H}$ [27]. Let $S \subset X$ be a complete countable type $\mathrm{II}_{\infty}$ section for the 
action of $H$. Then, by [21, Theorem 6.4], $R_{H} \approx \widetilde{R} \times I$, where $\widetilde{R}$ is the equivalence relation on $S$ with countable orbits (discrete reduction of type $\mathbb{I}_{\infty}$ ) and $I$ is the transitive equivalence relation generated by translation of the circle.

Let now $\theta$ be an automorphism of the equivalence relation $R_{H}$. According to [28, Theorem 2.4], the exist $\tilde{\theta} \in$ Aut $\widetilde{R}$ and $w \in \operatorname{Int} R_{H}$, such that $\theta=(\widetilde{\theta} \times \mathrm{id}) w$. The module of the automorphism $\theta$ is the number $\bmod \tilde{\theta}$, i.e. $\bmod \theta=\bmod \tilde{\theta}$ (see [28, Definition 2.9 and Remark 2.10]). Clearly, $\bmod \theta$ does not depend on the choice of the discrete reduction $\widetilde{R}$ and on the representation of $\theta$ as the product $(\tilde{\theta} \times \mathrm{id}) w$.

Definition B.1. The subgroup

$$
F\left(R_{H}\right)=\left\{\bmod \theta: \theta \in \text { Aut } R_{H}\right\}
$$

of the group $\boldsymbol{R}_{+}^{*}$ is called the fundamental group of the dynamical system $(H, X, \mu)$.

Theorem B.2. Let $H$ be a connected semisimple Lie group with a finite center, and let $(X, \mu)$ be a free ergodic $H$-space with finite invariant measure $\mu$. Suppose R-rank $(H) \geqslant 2$, and that the action $H$ on $X$ is irreducible, i.e. every simple factor of $H$ acts ergodically. Then, $F\left(R_{H}\right)=\{1\}$.

Proof. In view of [21, Lemma 6.9], the group $H$ may be thought to contain no compact factors and its center to be trivial.

Let $\theta$ be an outer automorphism $R_{H}$. Then,

$$
\theta(g x)=\alpha(g, x) \theta(x) \text { for all } g \in H \text { at a.a. } x \in X,
$$

where $\alpha: H \times X \rightarrow H$ is a Borel cocycle. The map $\theta: X \rightarrow X$ represent the orbit equivalence of the action on $X$ to itself. Hence, the Mackey action of thecocycle $\alpha$ is isomorphic to the said action [29, Proposition 2.5], which, as was demonstrated in [30], is Zariski dense in $H$. Thus, in view of [29, Theorem 4.1], there exists the Borel map $f: X \rightarrow X$ and the surjective endomorphism $\sigma: H \rightarrow H$, such that

$$
\alpha(g, x)=f(g x)^{-1} \sigma(g) f(x)
$$

where $\sigma$, by our assumptions on the group $H$, is an automorphism. Consider the Borel map

$$
\theta_{1}: X \rightarrow X, \quad \theta_{1}(x)=f(x) \theta(x) .
$$

From (B.1) and (B.2) it follows that

$$
\theta_{1}(g x)=\sigma(g) \theta_{1}(x) \quad \text { for all } \quad g \in H \text { at a.a. } x \in X .
$$


Hence follows

Lemma. The map $\theta_{1}$ is an automorphism of the space $(X, \mu)$ preserving measure $\mu$.

The proof used [31, Lemma 3.5].

Thus, the group $H$ and automorphism $\theta_{1}$ generate the locally compact group of transformations of the space $(X, \mu)$, i.e. the semidirect product $D=\boldsymbol{Z} \bigcirc_{\sigma} H$. Since any automorphism of the semisimple Lie group preserves its Haar measure, then $D$ is a unimodular group. Because, moreover, the action of $D$ on $X$ preserves the measure $\mu$, the equivalence relation $R_{D}$ is of type II. On the other hand, $\theta \theta_{1}^{-1} \in \operatorname{Int} R_{H}$, and thus the equivalence relation $R_{D}$ is isomorphic to the semidirect product $\boldsymbol{Z} \S_{\theta} R_{H}$ (see $[28, \S 2]$ ), i.e. $\boldsymbol{Z} \bigcirc_{\theta} R_{H}$ is a type II equivalence relation. Let now $\widetilde{R}$ be a type $\mathrm{II}_{\infty}$ discrete reduction for the equivalence relation $R_{H}$, i.e. $R_{H} \approx \widetilde{R} \times I$, and $\theta=(\widetilde{\theta} \times \mathrm{id}) w$ be the corresponding decomposition of the automorphism $\theta\left(\widetilde{\theta} \in\right.$ Aut $\left.\widetilde{R}, w \in \operatorname{Int} R_{H}\right)$ [28, Theorem 2.4]. Then,

$$
\boldsymbol{Z}\left(_{\theta} R_{H} \approx(\boldsymbol{Z} \subseteq \tilde{\theta} \widetilde{R}) \times I .\right.
$$

Hence, $\boldsymbol{Z}\left(S_{\tilde{\theta}} \widetilde{R}\right.$ is a type $I_{\infty}$ equivalence relation, and this means that $\bmod \tilde{\theta}=1$. Therefore, $\bmod \theta=1$, i.e. $F\left(R_{H}\right)=\{1\}$.

Corollary B.3. Let $\Gamma$ be a lattice in the connected simple Lie group $H$ with a finite center. Suppose R-rank $(H) \geqslant 2$. Let $(S, \mu)$ be a free ergodic $\Gamma$ space with the finite invariant measure $\mu$. Then, $F\left(R_{\Gamma}\right)=\{1\}$.

Proof. Consider the action of $H$ on the space $(X, \nu)$, induced by the action of $\Gamma$ on $(S, \mu)$ [26]. This action is free ergodic and preserves the measure $\nu$. Besides, $R_{H} \approx R_{\Gamma} \times I$. According to Theorem B.2, $F\left(R_{H}\right)=\{1\}$. Therefore, $F\left(R_{\Gamma}\right)=\{1\}$.

Remark B.4. Note that there exist countable ergodic equivalence relations with a unit fundamental group which do not have the property $T$ [27] (and therefore are not generated by the actions of lattices of Lie groups, as in Corollary B.3). Indeed, consider the group $\operatorname{SO}(n, \mathbb{Q})$ and its action by left shifts on $\mathrm{SO}(n, \boldsymbol{R}), n \geqslant 5$. The group $\mathrm{SO}(n, \boldsymbol{Q})$ can be represented as a countable union of a increasing chain of subgroups. Since this action is free, so is the corresponding equivalence relation, in contradiction to the property $\mathrm{T}[27,6.1 .4]$. However, as was shown in $\S 5$, the fundamental group of this action is trivial. 


\section{References}

[1] Murray, F.J. and Neumann, J. von, On rings of operators IV, Ann. Math., 44 (1943), 716-808.

[2] Kazhdan, D.A., Connection of the dual space of a group with the structure of its closed subgroups, Functional Anal. Appl., 1 (1967), 63-65.

[3] Connes, A., A factor fo type $\mathbb{I}_{1}$ with countable fundamental group, J. Operator Theory, 4 (1980), 151-153.

[4] Golodets, V. Ya. and Nessonov, N.I., T-Property and nonisomorphic full factors of types II and III, J. Funct. Anal., 70 (1987), 80-89.

[5] Golodets, V. Ya., Actions of $T$-groups on Lebesgue spaces and properties of full factors of type $\mathrm{II}_{1}, \quad$ Publ. RIMS, Kyoto Univ., 22 (1986), 613-636.

[6] Margulis, G.A., Some remarks of invariant means, Monatsh. Math., 80 (1980), 233235.

[7] Sullivan, D., For $n>3$ there is only one finitely additive rotationally invariant measure on the $n$-sphere defined on all Lebesgue measurable sets, Bull. Amer. Math. Soc., 4 (1981), 121-123.

[8] Gefter, S.L. and Golodets, V. Ya., Ergodic action with a unit fundamental group, (in Russian), Dokl. UkrSSR Acad. Sci., 10 (1986), 8-10.

[9] Gefter, S.L., Golodets, V. Ya. and Sinelshchikov, S.D., The fundamental group for ergodic actions of semisimple Lie groups and their lattices, (in Russian), Dokl.UkrSSR Acad. Sci., 8 (1987), 6-9.

[10] Feldman, J. and Moore, C.C., Ergodic equivalence relations, cohomology, and von Neumann algebras I, Trans. Amer. Math. Soc., 234 (1977), 289-324.

[11] — Ergodic equivalence relations, cohomology, and von Neumann algebras II, Trans. Amer. Math. Soc., 234 (1977), 325-359.

[12] Strătilă, S., Modular theory in operator algebras, (Ed. Academiei, Bucuresti, 1981.)

[13] Raghunathan, M.S., Discrete subgroups of Lie groups, (Springer-Verlag, Berlin-Heidelberg-New York, 1972).

[14] Choda, M., A condition to construct a full $\mathrm{II}_{1}$-factor with an application to approximate normalcy, Math. Jap., 28 (1983), 383-398.

[15] Haagerup, U., The standart form of von Neumann algebras, Math. Scand., 37 (1975), 271-283.

[16] Schmidt, K., Amenability, Kazhdan's property $T$, strong ergodicity and invariant means for ergodic group actions, Ergod. Theory and Dyn. Syst., $\mathbb{1}$ (1981), 223-236.

[17] Connes, A., Almost periodic states and factors of type $\mathrm{III}_{1}$, J. Funct. Anal., 16 (1974), 415-445.

[18] Choda, M., Crossed product and property T, Math. Jap., 26 (1981), 557-567.

[19] Diendonné, J., La géométrie des groupes classiques, (Springer-Verlag, Berlin-Heidelberg-New York, 1971).

[20] Golodets, V. Ya., The ergodic actions of $T$-groups, nonequivalent as to the trajectory, and the type $\mathrm{II}_{1}$ factors, (in Russian), Dokl. USSR Acad. Sci., 286 (1986), 524-527.

[21] Feldman, J., Hahn, P. and Moore, C.C., Orbit structure and countable sections for actions of continuous groups, Adv. in Math., 28 (1978), 186-230.

[22] Zimmer, R.J., Ergodic theory and semisimple groups, (Birkhäuser, Boston, 1984).

[23] Platonov, V.P., Arithmetical theory of algebraic groups, (in Russian), Uspekhi Mat. Nauk, 37 (1982), 3-54.

[24] Borel, A. and Harish-Chandra, Arithmetic subgroups of algebraic groups, Ann. of Math., 75 (1962), 485-535.

[25] Bourbaki, N., Groupes et algébres de Lie, (Hermann, Paris, 1971).

[26] Zimmer, R.J., Induced and amenable ergodic actions of Lie groups, Ann. Sci. Ecole Norm. Sup. (4), 11 (1978), 407-428. 
[27] Moore, C.C., Ergodic theory and von Neumann algebras, Proc. Symp. Pure Math., 38 (1982), part 2, 179-226.

[28] Golodets, V. Ya. and Sinelschhikov, S.D., Outer conjugacy for actions of continuous amenable groups, Publ. RIMS, Kyoto Univ., 23 (1987), 737-769.

[29] Zimmer, R.J., Strong rigidity for ergodic actions of semisimple Lie groups, Ann. of Math., 112 (1980), 511-529.

[30] — An algebraic group associated to an ergodic diffeomorphism, Compositio Math., 43 (1981), 59-69.

[31] — - Orbit equivalence and rigidity of ergodic actions of Lie groups, Ergod. Theory and Dyn. Syst., 1 (1981), 237-253. 
